Original Paper http://ajol.info/index.php/ijbcs http://indexmedicus.afro.who.int

\title{
Prévalence de la brucellose et de la tuberculose chez les bovins au Bénin
}

\author{
R. VIKOU ${ }^{1 *}$, L.G. APLOGAN ${ }^{2}$, C. AHANHANZO ${ }^{3}$, L. BABA-MOUSSA ${ }^{4}$ et \\ A.B. GBANGBOCHE ${ }^{1}$
}

'Laboratoire d'Amélioration Animale et de Biotechnologie Appliquée (LAABA), Institut des Sciences Biomédicales Appliquées (ISBA), Université d'Abomey-Calavi 01 BP : 526 Cotonou, Bénin.

${ }^{2}$ Laboratoire de Diagnostic Vétérinaire et Sérosurveillance des Maladies Animales, Ministère de l'Agriculture, de l'Elevage et de la Pêche, Bénin.

${ }^{3}$ Laboratoire de Génétique et des Biotechnologies (LGB), Département de Génétique et des Biotechnologies, Faculté des Sciences et Techniques (FAST), Université d'Abomey-Calavi 01 BP : 526 Cotonou, Bénin.

${ }^{4}$ Laboratoire de Biologie et de Typage Moléculaire en Microbiologie, Département de Biochimie et de Biologie Cellulaire, Faculté des Sciences et Techniques, Université d'Abomey-Calavi 05 BP 1604 Cotonou, Bénin.

*Auteur correspondant ; E-mail: vikouronaldess@yahoo.fr, Tel: +22996157243.

\section{REMERCIEMENTS}

Les auteurs remercient le Ministère de l'Enseignement Supérieur et de la Recherche Scientifique pour le support financier.

\section{RESUME}

Au Bénin, la production du lait occupe une place prépondérante dans l'économie des ménages ruraux. Elle fait souvent face à certaines maladies dont les conséquences sur la sécurité alimentaire et la santé publique sont énormes. C'est dans ce contexte que cette étude exploratoire a été conduite dans les communes de Parakou, Nikki, Tchaourou, Gogonou et Malanville, afin de déterminer chez les bovins, la prévalence de la brucellose et de la tuberculose. A cet effet, 525 bovins ont été prélevés et les sérums soumis au test ELISA indirect (enzyme- linked-immuno-sorbent- assay) pour la détection d'anticorps spécifique anti-brucella et les résultats obtenus révèlent 77 cas positifs (prévalence de 14,66\%). Pour le diagnostic de la tuberculose, 182 échantillons de laits prélevés chez 182 bovins ont été soumis à la coloration de Ziehl-Neelsen, donnant 67 cas positifs (prévalence de 36,81\%). La brucellose et la tuberculose étant deux zoonoses majeures pouvant porter atteinte à la santé des consommateurs, la prévalence de ces deux maladies est assez importante pour bénéficier de l'attention des producteurs, des consommateurs et de ceux qui interviennent dans la gestion de la santé animale.

(c) 2018 International Formulae Group. All rights reserved.

Mots clés: Prévalence, brucellose, tuberculose, bovins, Bénin. 


\title{
Prevalence of bovine brucellosis and tuberculosis in Benin
}

\begin{abstract}
In Benin, Milk production is a major factor in the economy of rural households. It often faces some diseases of which the consequences on food security and public health are numerous. It is in this context that this exploratory research was conducted in the communes of Parakou, Nikki, Tchaourou, Gogonou and Malanville, in order to determine in cattle, the prevalence of brucellosis and tuberculosis. To this end, 525 bovine animals were taken and serums subjected to the indirect ELISA (enzyme-linked-immuno-sorbent-assay) for the detection of specific antibodies against Brucella and the results obtained revealed 77 positive cases (prevalence of 14.66\%). For the diagnosis of tuberculosis, 182 samples of milk collected from 182 bovine animals were subjected to the staining of Ziehl-Neelsen, giving 67 positive cases (prevalence of $36.81 \%$ ). Since brucellosis and tuberculosis are two major zoonoses that can affect the health of consumers, the prevalence of these two diseases is important enough to benefit from the attention of producers, consumers and those who are involved in the management of animal health.
\end{abstract}

(C) 2018 International Formulae Group. All rights reserved.

Keywords: Prevalence, brucellosis, tuberculosis, bovine, Benin.

\section{INTRODUCTION}

La brucellose est une zoonose majeure, contagieuse, transmissible à de nombreuses espèces animales (Noudeke et al., 2017) et à l'homme (Ali et al., 2013 ; 2014). Elle est l'une des zoonoses les plus répandues dans le monde (Moreno, 2002 ; Noudeke et al., 2017) qui provoque de très importantes pertes économiques en élevage (Moreno, 2002) et représente un danger de santé publique non négligeable. Sa présence en élevage laitier est très préoccupante car le lait et ses dérivés constituent des sources majeures de contamination et de propagation des maladies. Chez les bovins, la brucellose est due, essentiellement à Brucella abortus. On distingue également Brucella melitensis, chez les moutons et les chèvres; Brucella suis chez les porcs; brucella ovis chez les agneaux, brebis et moutons; Brucella canis chez les chiens; Brucella neotoma, chez les rats du désert. Récemment, Brucella inopinata a été isolée chez les humains; Brucella. pinnipedialis et $B$. ceti chez les mammifères aquatiques et Brucella microti, chez les petits rongeurs (De figueiredo et al., 2015). Quant à la tuberculose, elle fait partie des maladies qui font payer un lourd tribu à la production de lait et de viande, elle est une maladie animale chronique principalement causée par la bactérie Mycobacterium bovis (M. bovis) qui est un membre du complexe M. tuberculosis. La maladie est caractérisée par le développement progressif de lésions granulomateuses ou de tubercules spécifiques dans le tissu pulmonaire, les nœuds lymphatiques ou d'autres organes (Bénet et al., 2014). Semblable à la forme humaine de la tuberculose, la tuberculose bovine attire de plus en plus l'attention de la communauté internationale en raison de l'augmentation importante du nombre de troupeaux infectés et des effets subséquents sur la production animale, combinés à l'impact significatif de $M$. bovis sur la santé publique, et à la menace permanente représentée par les animaux réservoirs à l'origine de l'infection (Thoen et al., 2009). Dans les pays moins développés, la tuberculose persiste chez les bovins et continue de causer des pertes importantes dans le secteur de l'élevage bovin, avec de graves conséquences pour la santé publique, en particulier dans les zones où la surveillance est inefficace et où les programmes de lutte sont faibles voire inexistants (Michel et al., 2010). Malheureusement la détermination de 
la prévalence réelle en Afrique subsaharienne des pathologies d'importance socioéconomique et d'impact sur la santé publique, est difficile à cause du manque de précision, de collaboration régionale, d'une faible représentativité des échantillons testés ou de l'inadéquation entre les résultats et les objectifs des études (Boukary et al., 2011). Au Bénin, il $\mathrm{y}$ a très peu d'informations précises sur l'importance de la brucellose, de la tuberculose et des infections intra mammaires. Le but de cette étude est de contribuer à améliorer l'état actuel des connaissances sur ces pathologies pour assurer effacement leur contrôle.

\section{MATERIEL ET METHODES Milieu d'étude}

Les études ont été conduites d'octobre 2015 à décembre 2015 en collaboration avec les chefs secteurs et les éleveurs. Douze fermes d'élevage dans les départements du Borgou et de l'Alibori au Nord du Bénin ont été ciblées au hasard (Figure 1). Le département de Borgou a un climat de type soudanien avec une seule saison des pluies. La pluviométrie annuelle varie entre 900 et 1300 mm par an. La saison des pluies commence en avril et dure sept mois environ. La température moyenne annuelle s'établit autour de $26{ }^{\circ} \mathrm{C}$ avec un maximum de $32{ }^{\circ} \mathrm{C}$ en mars et redescend aux environs de $23{ }^{\circ} \mathrm{C}$ en décembre-janvier. L'humidité relative varie entre 30 et $70 \%$ (INSAE, 2004a). Le climat du département de l'Alibori évolue du type soudanien dans sa partie Sud vers le type soudano-sahélien dans sa partie Nord (Karimama - Malanville). Elle ne connaît qu'une seule saison de pluie qui dure 5 à 6 mois avec une pluviosité oscillant entre 700 mm et $1200 \mathrm{~mm}$ (INSAE, 2004b)

\section{Prélèvements et constitution d'échantillons}

Les prélèvements ont été réalisés sur $10 \%$ des effectifs de bovin de toutes races confondues (Borgou, Yakana, Somba,
Goudali, Zébus et Métis divers (MAEP, 2002) dans chaque élevage visité. Au total 525 échantillons sanguins issus de 151 taureaux et 374 vaches en lactation et 394 échantillons de lait ont été prélevés. Ces échantillons ont été transportés au Laboratoire de Diagnostic Vétérinaire et de Séro-surveillance de Parakou pour les analyses.

\section{Diagnostic de la brucellose}

Pour la recherche de la brucellose, la technique ELISA indirect a été utilisée pour la détection des anticorps anti-Brucella abortus dans le sérum obtenu après centrifugation. A cet effet, ID Screen ${ }^{\circledR}$ Brucellosis Serum Indirect Multi-Species (société ID-vet) a été utilisé. Les sérums ont été regroupés en pouls de 6 sérums et testés en double pour la première étape de détection d'anticorps antiBrucella. Ensuite les pouls positifs $(0,110 \leq$ $\mathrm{DO} \geq 0,120$ ) ont été repris individuellement pour la confirmation des sérums positifs à la brucellose.

Les microplaques ont été présensibilisées avec du Lipopolysaccharide (LPS) de Brucella abortus. Les sérums à tester et les contrôles ont été dilués à $1 / 20$. A cet effet, $190 \mu \mathrm{l}$ de tampon de dilution ont été distribués dans les microplaques (cupules). Les cupules $\mathrm{A} 1$ et $\mathrm{B} 1$ ont été réservés aux contrôles négatifs et les cupules $\mathrm{C} 1$ et D1 aux contrôles positifs et ont reçu chacune $10 \mu \mathrm{lde}$ contrôle respectivement. Les cupules restantes ont également reçu chacune $10 \mu \mathrm{l}$ de sérum à tester. Les microplaques ont été ensuite incubées 45 minutes à $21^{\circ} \mathrm{C}$ puis laver trois fois avec environ $300 \mu \mathrm{l}$ de solution de lavage en évitant le dessèchement des cupules entre les lavages. Pendant cette phase, les anticorps anti-Brucella, s'ils sont présents, forment un complexe antigène-anticorps. 100 $\mu 1$ de conjugué multi-espèce marqué à la peroxydase (HRP) ont été distribués dans les cupules ou microplaques puis incuber 30 minutes à $21{ }^{\circ} \mathrm{C}$ puis laver trois fois avec environ $300 \mu \mathrm{l}$ de Solution de lavage. Il se 
fixe aux anticorps anti-Brucella, formant un complexe antigène anticorps-conjugué-HRP. Après élimination du conjugué en excès par lavage, la réaction a été révélée par $100 \mu \mathrm{l}$ de solution de révélation (TMB) dans chaque cupule puis incuber 15 minutes à $21^{\circ} \mathrm{C}$ à l'obscurité. Enfin, $100 \mu \mathrm{l}$ de solution d'arrêt ont été distribuées dans chaque cupule pour arrêter la réaction. En présence d'anticorps dans l'échantillon, il apparaît une coloration bleue qui devient jaune après blocage et en l'absence d'anticorps dans l'échantillon, il n'apparaît pas de coloration. Les microplaques ont été lues et enregistrées à $450 \mathrm{~nm}$ à l'aide d'un lecteur de Marque 'Biotek'. Le test a été validé si la valeur moyenne de densité optique des contrôles positifs (DOcp) est supérieure à 0,350 et si le rapport entre la moyenne des contrôles positifs (DOcp) et la moyenne des Contrôles Négatifs (DOcn) est supérieur à 3. Les échantillons présentant un pourcentage (S/P) inférieur ou égal à $110 \%$ ont été considérés comme négatifs, supérieur à $110 \%$ et inférieur à $120 \%$ ont été considérés comme douteux et supérieur ou égal à $120 \%$ ont été considérés comme positifs.

\section{Diagnostic de la tuberculose}

Les crèmes obtenus après centrifugation des laits ont subi les différentes étapes de la coloration de Ziehl-Neelsen pour la détection des bacilles. Elle est composée principalement de la solution de fuchsine, solution de bleu de méthylène et de l'acide nitrique selon la procédure de Ziehl-Neelsen (Warren et al., 2000). Pour la recherche de la tuberculose, chaque lait recueilli a été soumis aux trois grandes étapes de la coloration de Ziehl-Neelsen qui est une méthode de coloration permettant l'identification des mycobactéries au microscope. Elle fait partie des colorations qui mettent en évidence l'acido-alcoolo-résistance, caractère fondamental des mycobactéries, en prenant en compte la difficulté de pénétration des colorants.

Pour la coloration, Chaque frottis a été recouvert d'un papier filtre imprégné de fuchsine de Ziehl puis chauffé pendant dix minutes au moins à partir d'une émission de vapeur, en veillant à ne pas laisser sécher le papier, suivi d'un rinçage à l'eau distillée stérile. Ensuite, pour la décoloration, la lame a été trempée dans une solution d'acide pendant deux minutes, suivi d'un rinçage et après trempée pendant cinq minutes dans l'alcool à $90^{\circ}$ puis rincée. Enfin, pour recolorer, la lame a été recouverte d'une solution de bleu de méthylène pendant deux minutes, puis rincée. Les observations microscopes ont été effectuées avec objectif à immersion et les mycobactéries apparaissent alors comme des bacilles rouges sur fond bleu-gris en cas de tuberculose.

\section{Analyses statistiques}

Pour chaque échantillon, le pourcentage $\mathrm{S} / \mathrm{P} \quad(\mathrm{S} / \mathrm{P} \%)$ a été calculé en utilisant cette formule: $\mathrm{S} / \mathrm{P} \%=\mathrm{DO}$ échantillon - Docn / DOcp - Docn x100. DO échantillon étant la densité optique de chaque échantillon, DOcn étant la densité du contrôle négatif et DOcp étant celle du contrôle positif. La prévalence globale pour chaque infection a été calculée en faisant le rapport entre le nombre total de cas positifs et l'effectif total. Ainsi, la prévalence de la brucellose par commune et par sexe a été calculée en faisant le rapport de nombre de cas positifs par commune et par sexe, de même que celle de la tuberculose. 


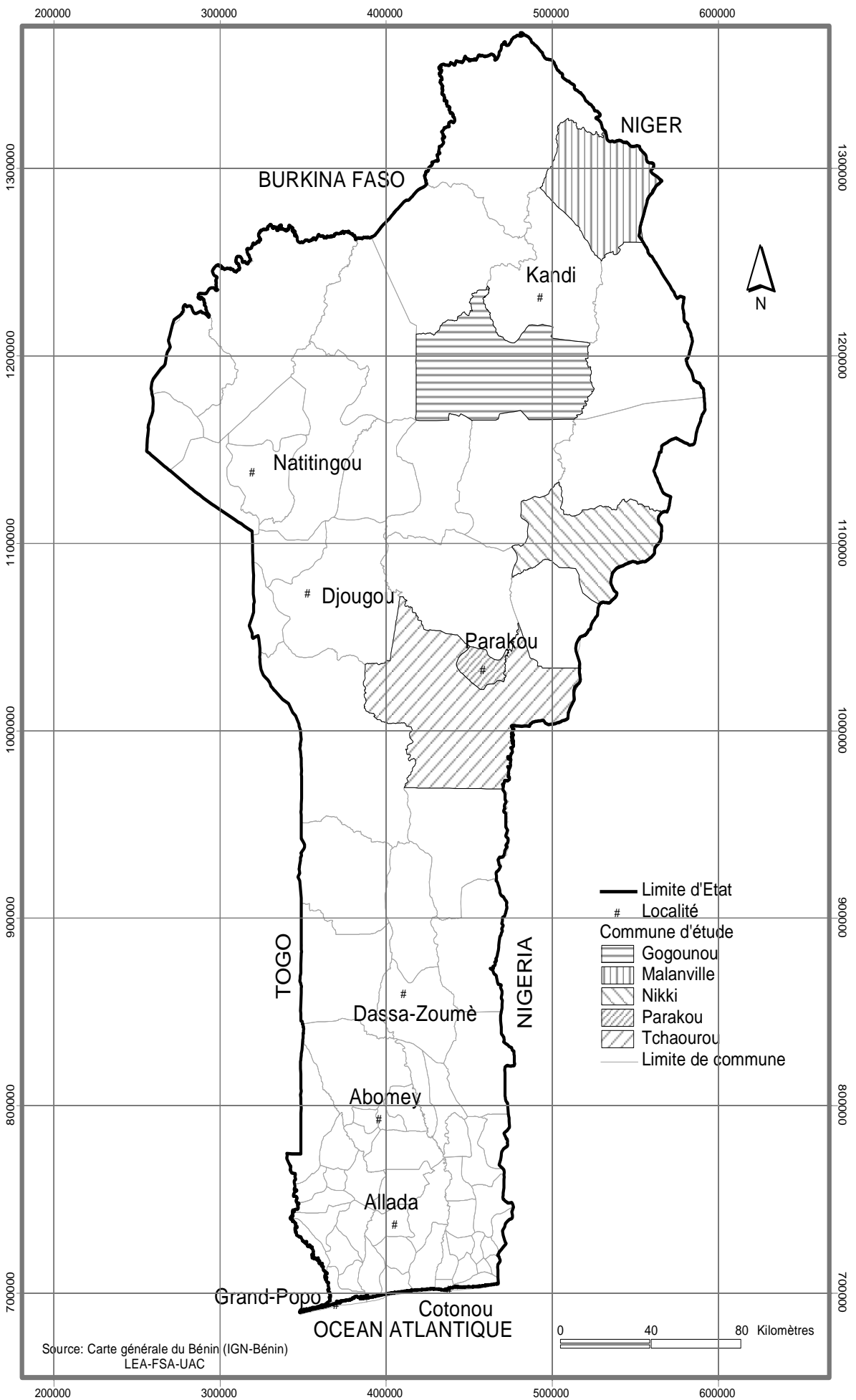

Figure 1: Zone d'étude de la brucellose et la tuberculose au Bénin. 


\section{RESULTATS}

Les résultats traduisant la distribution de la prévalence de la brucellose et la tuberculose sont respectivement dans les Tableaux 1 et 2 .

Les résultats du Tableau 1 montrent que femelles sont plus infectées par Brucella abortus et son absence dans la commune de Gogounou. On observe de faible prévalence de la brucellose dans les communes de Parakou (8\%) et de Nikki $(6,6 \%)$ contrairement aux communes de Tchaourou $(35 \%)$ et de Malanville (31\%) où elles sont élevées.

La prévalence de la brucellose révèle 77 cas liés à la présence d'anticorps antiBrucella. Plus élevée chez les femelles que les mâles et une forte concentration est observée dans les localités de Tchaourou et de Malanville, viennent après Parakou et Nikki (Tableau 1). La prévalence de la tuberculose (Tableau 2) est plus marquée à Tchaourou et régresse progressivement de Parakou à Malanville et à Nikki. Elle révèle 67 cas positifs soit une prévalence globale de $36,8 \%$ de tuberculose dans les élevages de l'étude. Les résultats du Tableau 2 montrent l'absence de la tuberculose de la commune de Gogounou, de forte présence dans la commune de Tchaourou (68\%). Elle est également présente dans les communes de Parakou (41,6\%), de Nikki $(26,5 \%)$ et de Malanville (37,2\%).

Tableau 1 : Distribution des prévalences de la brucellose selon les élevages et leur regroupement par localité.

\begin{tabular}{|c|c|c|c|c|c|c|c|c|c|c|}
\hline \multirow[t]{2}{*}{ Localités } & \multirow[t]{2}{*}{ Elevages } & \multirow[t]{2}{*}{$\begin{array}{l}\text { Effectif } \\
\text { (Sérums) }\end{array}$} & \multicolumn{2}{|c|}{$\begin{array}{l}\text { Animaux } \\
\text { prélevés }\end{array}$} & \multicolumn{2}{|c|}{ Animaux positifs } & \multicolumn{2}{|c|}{ Prévalence (\%) } & \multicolumn{2}{|c|}{$\begin{array}{l}\text { Prévalence } \\
\text { globale (\%) }\end{array}$} \\
\hline & & & Mâle & Femelle & Mâle & Femelle & Mâle & Femelle & Mâle & Femelle \\
\hline \multirow{3}{*}{ Tchaourou } & Guinero1 & \multirow{3}{*}{104} & 9 & 41 & 0 & 18 & 0 & 43,9 & & \\
\hline & Guinero2 & & 4 & 50 & 0 & 14 & 0 & 28 & 0 & 35 \\
\hline & Sirarou & & 12 & 46 & 1 & 6 & 8 & 13 & & \\
\hline \multirow[t]{2}{*}{ Parakou } & Darnon & 103 & 7 & 38 & 0 & 1 & 0 & 2,6 & 5,26 & 8 \\
\hline & Tchekenté & & 21 & 52 & 0 & 2 & 0 & 3,8 & & \\
\hline \multirow[t]{2}{*}{ Nikki } & Koussoukou & 104 & 8 & 23 & 0 & 3 & 0 & 13 & 0 & 6,6 \\
\hline & Wolobanga & & 6 & 29 & 0 & 20 & 0 & 19 & & \\
\hline \multirow[t]{2}{*}{ Malanville } & Banga & 112 & 3 & 74 & 0 & 12 & 0 & 11,6 & 0 & 31 \\
\hline & Zoukarou1 & & 27 & 5 & 0 & 0 & 0 & 0 & & \\
\hline Gogounou & Zoukarou 2 & 102 & 54 & 16 & 0 & 0 & 0 & 0 & 0 & 0 \\
\hline Total & 10 & 525 & 151 & 374 & 1 & 76 & 0,66 & 20,3 & \multicolumn{2}{|c|}{14,66} \\
\hline
\end{tabular}


Tableau 2: Distribution des prévalences de la tuberculose selon les élevages et leur regroupement par localité.

\begin{tabular}{lccccc}
\hline Localités & Elevages & Effectif (Laits) & \multicolumn{2}{c}{ Animaux } & Prévalence (\%) \\
\hline \multirow{2}{*}{ Tchaourou } & Guinero1 & & Négatifs & Positifs & \\
& & 19 & 0 & 8 & 68 \\
& Guinero2 & & 6 & 5 & \\
\multirow{2}{*}{ Parakou } & Sirarou & & 8 & 5 & 41,6 \\
& Darnon & 24 & 6 & 5 & 26,5 \\
Nikki & Tchekenté & & 13 & 6 & \\
& koussoukou & 34 & 12 & 3 & 37,2 \\
Malanville & Wolobanga & & 13 & 8 & \\
& Banga & 94 & 46 & 27 & 0 \\
Gogounou & Zoukarou1 & & 4 & 0 & 36,8 \\
Total & Zoukarou 2 & 11 & 7 & 0 & \\
\hline
\end{tabular}

\section{DISCUSSION}

L'évaluation du taux de prévalence de la brucellose et de la tuberculose a été déterminée par des tests indirects à savoir l'ELISA indirecte pour la brucellose et la coloration de Ziehl-Neelsen pour la tuberculose. Les tests immuno-enzymatiques ELISA sont connus comme des tests sérologiques très sensibles détectant aussi bien les infections récentes qu'anciennes et chroniques. Ainsi la prévalence globale de la brucellose de 14,66\% obtenue confirme la persistance de la maladie à l'Okpara, Bétécoucou, Samiondji et Kpinnou comme signalée par Koutinhouin et al. (2003) avec une prévalence de $15,21 \%$ en utilisant le même test sérologique. Les résultats confirment les travaux de Noudeke et al. (2017) qui signalaient aussi l'infection à brucellose dans le Nord du Bénin dans la période d'octobre à décembre en utilisant également le même test sérologique. Néanmoins, il aurait été intéressant aussi d'associer la réaction au Rose Bengale ou l'épreuve à l'antigène tampon (EAT) qui est aussi sensible et dont l'indice de performance est le plus élevé (IP = 193,1) que les autres méthodes pour confirmer la brucellose selon Gall et Nielsen, 2004. Sa sensibilité est estimée entre $91,4 \%$ et $100 \%$ et sa spécificité est supérieure à $99 \%$ en zone indemne et comprise entre $95 \%$ et $99 \%$ dans les régions où il $\mathrm{y}$ a une forte prévalence de réactions non spécifiques sur des bovins (Boussini et al., 2012). Pour la tuberculose, on pourrait aussi utiliser l'intradermotuberculination simple (IDS), une méthode universelle fiable le plus utilisée (70\% des cas) pour le dépistage de la tuberculose chez les bovins (OMS, 2004; Ameni et al., 2007).

\section{Conclusion}

Cette étude a permis de mettre en évidence l'existence de la brucellose et de la tuberculose dans les troupeaux bovins des élevages au Nord Bénin avec une prévalence globale de $14,66 \%$ pour la brucellose et $36,8 \%$ pour la tuberculose. Mais il serait nécessaire de confirmer ces résultats par l'identification des bactéries, à partir de lésions, sur les bovins à l'abattoir pour la tuberculose, ou lors d'avortements dans les élevages pour la brucellose.

\section{CONFLITS D'INTERETS}

Les auteurs déclarent qu'il n'y a aucun conflit d'intérêts en lien avec cet article.

\section{CONTRIBUTIONS DES AUTEURS}

$\mathrm{RV}$ a participé à toutes les phases du travail : collecte des échantillons, analyse de laboratoire et rédaction. LGA a contribué à l'analyse des échantillons. CA et JB-M ont travaillé sur le protocole exécuté sur le terrain 
et la relecture du projet d'article. ABG a supervisé les travaux de collecte et l'analyse des échantillons au laboratoire, assuré l'analyse statistique des données et de leur interprétation.

\section{REFERENCES}

Ali S, Ali Q, Melzer F, Khan I, Akhter S, Neubauer H, Jamal SM. 2014. Isolation and identification of bovine Brucella isolates from Pakistan by biochemical tests and PCR. Trop Anim. Health Prod., 46: 73-78. DOI: 10.1007/s11250-0130448-6.

Ali S, Ali Q, Neubauer H, Melzer F, Elschner M, Khan I, Abatih EN, Ullah N, Irfan M, Akhter S. 2013. Seroprevalence and Risk Factors Associated with Brucellosis as a Professional Hazard in Pakistan. Foodborne Pathog Dis., 10: 500505.DOI: 10.1089/fpd.2012.1360

Ameni G, Aseffa A, Sirak A, Engers H, Young DB, Hewinson GR, Vordermeier MH, Gordon SV. 2007. Effect of skin testing and segregation on the incidence of Bovine tuberculosis, and molecular typing of Mycobacterium bovis in Ethiopia. Vet Rec., 161: 782-786. https://www.ncbi.nlm.nih.gov/pmc/articl es/PMC2292248/

Bénet JJ, Praud A, 2014. La tuberculose animale. Polycopié des Unités de maladies contagieuses des Ecoles Nationales Vétérinaires françaises, Mérial (Lyon), $100 \mathrm{p}$.

Boukary AR, Thys E, Mamadou S, Rigouts L, Matthys F, Vias Franck SG, Gamatie D, Yenikoye A, Saegerman C. 2011. La tuberculose à Mycobacterium bovis en Afrique subsaharienne. Ann. Méd. Vét., 155 : 23-37.

Boussini H, Traoré A, Tamboura HH, Bessin R, Boly H, Ouédraogo A. 2012. Prévalence de la tuberculose et de la brucellose dans les élevages bovins laitiers intra-urbains et périurbains de la ville d'Ouagadougou au Burkina Faso. Rev. Sci. Tech. Off. Int. Epiz., 31(3): 943-951.
De figueiredo $\mathrm{P}$, Thomas AF, Allison RF, Carlos AR, Garry AL. 2015. Pathogenesis and Immunobiology of Brucellosis Review of Brucella-Host Interactions. Am. J. Pathol., 185: 15051517. DOI: http://dx.doi.org/10.1016/ j.ajpath.2015.03.003

Gall D, Nielsen K. 2004. Comparaison des méthodes sérologiques de diagnostic de la brucellose bovine en termes de performances et de coûts. Rev. Sci. Tech. Off. Int. Epiz., 23(3): 980-1002.

Ibrahim N, Belihu K, Lobago F, Bekena M. 2010. Sero-Prevalence Of Bovine Brucellosis And Risk Factors In Jimma Zone Of Oromia Region, South-Western Ethiopia. Trop. Anim. Health Prod., 42, 35-40. DOI 10.1007/s11250-009-9382-z.

INSAE (Institut National de la Statistique et de l'Analyse Economique). 2004a. Rgph3. Cahier Des Villages Et Quartiers De Ville Département Du Borgou.www.insae-bj.org/recensementpopulation.html?.../borgou Consulté le 18/9/2017

INSAE (Institut National de la Statistique et de l'Analyse Economique). 2004b. Rgph3. Cahier Des Villages Et Quartiers De Ville Département De L'alibori.www.insae-

Bj.Org/Recensement-

Population.Html?.../Alibori. Consulté le 18/9/2017

Koutinhouin B, Youssao AKI, Houehou AE, Agbadje PM. 2003. Prévalence de La Brucellose Bovine Dans Les Elevages Traditionnels Encadrés Par Le Projet Pour Le Développement De l'Elevage (PDE) $\mathrm{Au}$ Bénin. Revue. Méd. Vét., 154(4): 271-276. DOI : https://doi.org/10.5897/JVMAH2017.05 67

MAEP (Ministère de L'agriculture, de L'élevage et de la Pèche). 2002. Rapport national sur l'état des ressources zootechniques. Direction de l'Elevage. www.fao.org/AG/againfo/programmes/e n/genetics/.../Benin.pdf. Consulté le $2 / 03 / 2018$ 
Michel AL, Müller B, van Helden PD. 2010. Mycobacterium bovis at the animalhuman interface: a problem, or not? Vet. Microbiol., 140(3-4): 371-381. DOI: 10.1016/j.vetmic.2009.08.029

Moreno E. 2002. Brucellosis in Central America. Vet. Microbiol., 90: 31-38. DOI : $\quad 10.1016 / S 0378-1135(02)$ 002420 .

Noudeke ND, Aplogan LG, Dossa F, Youssao I, Farougou S. 2017. Monthly variations of the prevalence of bovine brucellosis in Benin. Adv. Anim. Vet. Sci., 5(1): 2329.DOI: http://dx.doi.org/10.14737/ journal.aavs/2017/5.1.23.29.

Thoen CO, LoBue P, Enarson DA, Kaneene JB, de Kantor IN. 2009. Tuberculosis: a re-emerging disease of animals and humans. Veterinaria Italiana, 45(1): 135-181.

OMS (Organisation Mondiale de la Santé). 2004. World Health Organization Office for the African Region, Harare, Zimbabwe October 2004TB/HIV Control Strategy for the African Region, $22 \mathrm{p}$.

Warren JR, Bhattacharya M, De Almeida KNF, Trakas K, Peterson LR. 2000. A Minimum 5.0ml of Sputum Improves the Sensitivity of Acid-fast Smear for Mycobacterium tuberculosis. Am. J. Respir. Crit. Care Med., 161: 15591562. DOI: $10.1164 /$ ajrccm.161.5. 9908063 . 